EPJ Web of Conferences 69, 00015 (2014)

DOI: $10.1051 /$ epjconf / 20146900015

(C) Owned by the authors, published by EDP Sciences, 2014

\title{
Fusion at near-barrier energies within the quantum diffusion ap- proach
}

\author{
V.V.Sargsyan ${ }^{1,2}$, G.G.Adamian ${ }^{1, a}$, N.V.Antonenko ${ }^{1}$, W.Scheid ${ }^{3}$, and H.Q.Zhang ${ }^{4}$ \\ ${ }^{1}$ Joint Institute for Nuclear Research, 141980 Dubna, Russia \\ ${ }^{2}$ International Center for Advanced Studies, Yerevan State University, 0025 Yerevan, Armenia \\ ${ }^{3}$ Institut für Theoretische Physik der Justus-Liebig-Universität, D-35392 Giessen, Germany \\ ${ }^{4}$ China Institute of Atomic Energy, Post Office Box 275, Beijing 102413, China
}

\begin{abstract}
Within the quantum diffusion approach, the role of neutron transfer in fusion (capture) reactions with tightly and weakly bound nuclei is discussed. The breakup process is analyzed. New methods for the study of the breakup probability are suggested.
\end{abstract}

\section{Introduction}

The nuclear deformation and neutron-transfer process have been identified as playing a major role in the magnitude of the sub-barrier fusion (capture) cross sections [1]. There are several experimental evidences which confirm the importance of nuclear deformation on the fusion. The influence of nuclear deformation is straightforward. If the target nucleus is prolate in the ground state, the Coulomb field on its tips is lower than on its sides, which then increases the capture or fusion probability at energies below the barrier corresponding to the spherical nuclei. The role of neutron transfer reactions is less clear. The importance of neutron transfer with positive $Q$-values on nuclear fusion (capture) originates from the fact that neutrons are insensitive to the Coulomb barrier and they can therefore start being transferred at larger separations before the projectile is captured by the target nucleus. Therefore, it is generally thought that the sub-barrier fusion cross section will increase because of neutron transfer.

The fusion (capture) dynamics induced by loosely bound radioactive ion beams is currently being extensively studied. However, the long-standing question whether fusion (capture) is enhanced or suppressed with these beams has not yet been answered unambiguously. The study of fusion reactions involving nuclei at the drip-lines has led to contradictory results.

\section{Quantum diffusion approach for capture}

In the quantum diffusion approach [2-6] the capture of the projectile by the target-nucleus is described with a single relevant collective variable: the relative distance between the colliding nuclei. This approach takes into consideration the fluctuation and dissipation effects in collisions of heavy ions that model the coupling of the relative motion with various channels (for example, the non-collective

\footnotetext{
ae-mail: adamian@theor.jinr.ru
} 
single-particle excitations, low-lying collective dynamical modes of the target and projectile). The nuclear static deformation effects are taken into account through the dependence of the nucleus-nucleus potential on the deformations and mutual orientations of the colliding nuclei. We have to mention that many quantum-mechanical and non-Markovian effects accompanying the passage through the potential barrier are taken into consideration in our formalism [2, 4].

The capture cross section is a sum of partial capture cross sections $[2,4]$

$$
\begin{aligned}
\sigma_{c a p}\left(E_{\mathrm{c} . \mathrm{m} .}\right) & =\sum_{J} \sigma_{\text {cap }}\left(E_{\mathrm{c} . \mathrm{m} .}, J\right)= \\
& =\pi \lambda^{2} \sum_{J}(2 J+1) \int_{0}^{\pi / 2} d \theta_{1} \sin \left(\theta_{1}\right) \int_{0}^{\pi / 2} d \theta_{2} \sin \left(\theta_{2}\right) P_{\text {cap }}\left(E_{\text {c.m. }}, J, \theta_{1}, \theta_{2}\right),
\end{aligned}
$$

where $\lambda^{2}=\hbar^{2} /\left(2 \mu E_{\text {c.m. }}\right)$ is the reduced de Broglie wavelength, $\mu=m_{0} A_{1} A_{2} /\left(A_{1}+A_{2}\right)$ is the reduced mass ( $m_{0}$ is the nucleon mass), and the summation is over the possible values of the angular momentum $J$ at a given bombarding energy $E_{\text {c.m. }}$. Knowing the potential of the interacting nuclei for each orientation with the angles $\theta_{i}(i=1,2)$, one can obtain the partial capture probability $P_{\text {cap }}$ which is defined by the probability of passing through the potential barrier in the relative distance $R$ coordinate at a given $J$. The value of $P_{\text {cap }}$ is obtained by integrating the propagator $G$ from the initial state $\left(R_{0}, P_{0}\right)$ at time $t=0$ to the final state $(R, P)$ at time $t$ ( $P$ is a momentum):

$$
P_{\text {cap }}=\lim _{t \rightarrow \infty} \int_{-\infty}^{r_{\text {in }}} d R \int_{-\infty}^{\infty} d P G\left(R, P, t \mid R_{0}, P_{0}, 0\right)=\lim _{t \rightarrow \infty} \frac{1}{2} \operatorname{erfc}\left[\frac{-r_{\text {in }}+\overline{R(t)}}{\sqrt{\Sigma_{R R}(t)}}\right] .
$$

The second line in (2) is obtained by using the propagator $G=\pi^{-1}\left|\operatorname{det} \boldsymbol{\Sigma}^{-1}\right|^{1 / 2} \exp \left(-\mathbf{q}^{T} \boldsymbol{\Sigma}^{-1} \mathbf{q}\right)\left(\mathbf{q}^{T}=\right.$ $\left[q_{R}, q_{P}\right], q_{R}(t)=R-\overline{R(t)}, q_{P}(t)=P-\overline{P(t)}, \overline{R(t=0)}=R_{0}, \overline{P(t=0)}=P_{0}, \Sigma_{k k^{\prime}}(t)=2 \overline{q_{k}(t) q_{k^{\prime}}(t)}$, $\left.\Sigma_{k k^{\prime}}(t=0)=0, k, k^{\prime}=R, P\right)$ calculated for an inverted oscillator that approximates the nucleusnucleus potential $V$ in the variable $R$. The frequency $\omega$ of this oscillator with an internal turning point $r_{\text {in }}$ is defined from the condition of equality of the classical actions of approximate and realistic potential barriers of the same hight at given $J$. This approximation is well justified for the reactions and energy range that are here considered.

We assume that the sub-barrier capture mainly depends on the optimal one-neutron $\left(Q_{1 n}>Q_{2 n}\right)$ or two-neutron $\left(Q_{2 n}>Q_{1 n}\right)$ transfer with positive $Q$-value. Our assumption is that, just before the projectile is captured by the target-nucleus (just before crossing the Coulomb barrier), which is a slow process, the transfer occurs and can lead to the population of the first excited collective state in the recipient nucleus [7] (the donor nucleus remains in the ground state). So, the motion to $N / Z$ equilibrium starts in the system before the capture because it is energetically favorable in the dinuclear system in the vicinity of the Coulomb barrier. For the reactions under consideration, the average change of mass asymmetry is connected to the one- or two-neutron transfer ( $1 n$ - or $2 n$-transfer). Since after the transfer the mass numbers, the isotopic composition and the deformation parameters of the interacting nuclei, and, correspondingly, the height $V_{b}=V\left(R_{b}\right)$ and shape of the Coulomb barrier are changed, one can expect an enhancement or suppression of the capture. If after the neutron transfer the deformations of interacting nuclei increase (decrease), the capture probability increases (decreases). When the isotopic dependence of the nucleus-nucleus potential is weak and the deformations of the interacting nuclei do not change after the transfer, there is no effect of the neutron transfer on the capture. In comparison with Ref. [8], we assume that the negative transfer $Q$-values do not play a visible role in the capture process. Our scenario was verified in the description of many reactions [4$6]$. 


\section{$\mathrm{CNR} * 13$}

\section{Results of calculations}

Because the capture cross section is equal to the complete fusion cross section for the reactions treated, the quantum diffusion approach for capture is applied to study the complete fusion. All calculated results are obtained with the same set of parameters as in Ref. [2]. A realistic friction coefficient in the relative distance coordinate $\hbar \lambda=2 \mathrm{MeV}$ is used. Its value is close to that calculated within mean-field approaches [9]. For the nuclear part of the nucleus-nucleus potential, the double-folding formalism with the Skyrme-type density-dependent effective nucleon-nucleon interaction is used [2,4]. The parameters of the nucleus-nucleus interaction potential $V(R)$ are adjusted to describe the experimental data at energies above the Coulomb barrier corresponding to spherical nuclei. The absolute values of the experimental quadrupole deformation parameters $\beta_{2}$ of even-even deformed nuclei in the ground state and of the first excited collective states of nuclei are taken from Ref. [10]. For the nuclei deformed in the ground state, the $\beta_{2}$ of the first excited collective state is similar to the $\beta_{2}$ of the ground state. For the quadruple deformation parameter of an odd nucleus, we choose the maximal value from the deformation parameters of neighboring even-even nuclei (for example, $\left.\beta_{2}\left({ }^{231} \mathrm{Th}\right)=\beta_{2}\left({ }^{233} \mathrm{Th}\right)=\beta_{2}\left({ }^{232} \mathrm{Th}\right)=0.261\right)$. For the double magic and neighboring nuclei, we take $\beta_{2}=0$ in the ground state. Since there are uncertainties in the definition of the values of $\beta_{2}$ in light-mass nuclei, one can extract the ground-state quadrupole deformation parameters of these nuclei from a comparison of the calculated capture cross sections with the existing experimental data. By describing the reactions ${ }^{12} \mathrm{C}+{ }^{208} \mathrm{~Pb},{ }^{18} \mathrm{O}+{ }^{208} \mathrm{~Pb},{ }^{32,36} \mathrm{~S}+{ }^{90} \mathrm{Zr},{ }^{34} \mathrm{~S}+{ }^{168} \mathrm{Er},{ }^{36} \mathrm{~S}+{ }^{90,96} \mathrm{Zr},{ }^{58} \mathrm{Ni}+{ }^{58} \mathrm{Ni}$, and ${ }^{64} \mathrm{Ni}+{ }^{58} \mathrm{Ni}$, where there are no neutron transfer channels with positive $Q$-values, we extract the ground-state quadrupole deformation parameters $\beta_{2}=-0.3,0.1,0.312,0.1,0,0.05$, and 0.087 , for the nuclei ${ }^{12} \mathrm{C},{ }^{18} \mathrm{O},{ }^{32} \mathrm{~S},{ }^{34} \mathrm{~S},{ }^{36} \mathrm{~S},{ }^{58} \mathrm{Ni}$, and ${ }^{64} \mathrm{Ni}$, respectively, which are used in our calculations.

\subsection{Role of neutron transfer in capture process at sub-barrier energies}

After the neutron transfer in the reaction ${ }^{40} \mathrm{Ca}\left(\beta_{2}=0\right)+{ }^{96} \mathrm{Zr}\left(\beta_{2}=0.08\right) \rightarrow{ }^{42} \mathrm{Ca}\left(\beta_{2}=0.247\right)+$ ${ }^{94} \operatorname{Zr}\left(\beta_{2}=0.09\right)$ (Fig. 1) or ${ }^{40} \mathrm{Ca}\left(\beta_{2}=0\right)+{ }^{124} \mathrm{Sn}\left(\beta_{2}=0.095\right) \rightarrow{ }^{42} \mathrm{Ca}\left(\beta_{2}=0.247\right)+{ }^{122} \mathrm{Sn}\left(\beta_{2}=0.1\right)$ (Fig. 1) the deformation of the nuclei increases and the mass asymmetry of the system decreases. Thus, the value of the Coulomb barrier decreases and the capture cross section becomes larger (Fig. 1). In Fig. 2, we observe the same behavior in the reactions ${ }^{58} \mathrm{Ni}\left(\beta_{2}=0.05\right)+{ }^{132} \mathrm{Sn}\left(\beta_{2}=0\right) \rightarrow{ }^{60} \mathrm{Ni}\left(\beta_{2}=\right.$ $0.207)+{ }^{130} \mathrm{Sn}\left(\beta_{2}=0\right)\left(Q_{2 n}=7.8 \mathrm{MeV}\right),{ }^{58} \mathrm{Ni}\left(\beta_{2}=0.05\right)+{ }^{130} \mathrm{Te}\left(\beta_{2}=0\right) \rightarrow{ }^{60} \mathrm{Ni}\left(\beta_{2}=0.207\right)+$ ${ }^{128} \mathrm{Te}\left(\beta_{2}=0\right)\left(Q_{2 n}=5.9 \mathrm{MeV}\right),{ }^{64} \mathrm{Ni}\left(\beta_{2}=0.087\right)+{ }^{132} \mathrm{Sn}\left(\beta_{2}=0\right) \rightarrow{ }^{66} \mathrm{Ni}\left(\beta_{2}=0.158\right)+{ }^{130} \mathrm{Sn}\left(\beta_{2}=0\right)$ $\left(Q_{2 n}=2.5 \mathrm{MeV}\right)$, and ${ }^{64} \mathrm{Ni}\left(\beta_{2}=0.087\right)+{ }^{130} \mathrm{Te}\left(\beta_{2}=0\right) \rightarrow{ }^{66} \mathrm{Ni}\left(\beta_{2}=0.158\right)+{ }^{128} \mathrm{Te}\left(\beta_{2}=0\right)$ $\left(Q_{2 n}=0.55 \mathrm{MeV}\right)$. One can see a good agreement between the calculated results and the experimental data [11-13]. So, the observed capture enhancement at sub-barrier energies in the reactions mentioned above is related to the two-neutron transfer channel. One can see that at energies above and near the Coulomb barrier the cross sections with and without two-neutron transfer are almost similar. Since the two-neutron transfer causes a larger change of the deformations of the nuclei in the reactions ${ }^{58} \mathrm{Ni}$ $+{ }^{132} \mathrm{Sn},{ }^{130} \mathrm{Te}$ than in the reactions ${ }^{64} \mathrm{Ni}+{ }^{132} \mathrm{Sn},{ }^{130} \mathrm{Te}$, at sub-barrier energies the capture enhancement in the reactions with ${ }^{58} \mathrm{Ni}$ is larger than in the reactions with ${ }^{64} \mathrm{Ni}$ (Fig. 2).

One can make unambiguous statements regarding the neutron transfer process with a positive $Q$-value when the colliding nuclei are double magic or semi-magic. In this case one can disregard the deformation and orientation effects before the neutron transfer. To eliminate the influence of the nucleus-nucleus potential on the capture (fusion) cross section and to make conclusions about the role of deformation of colliding nuclei and the nucleon transfer between interacting nuclei in the capture (fusion) cross section, a reduction procedure is useful [15]. It consists of the following 

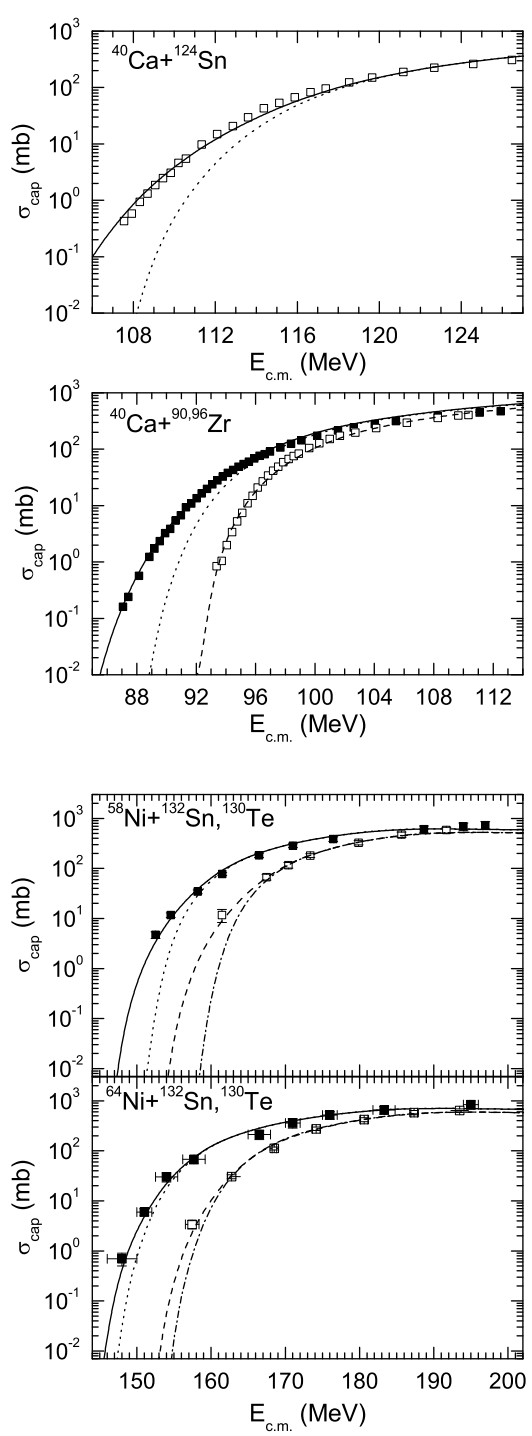

Figure 1. The calculated capture cross sections versus $E_{\text {c.m. }}$ for the indicated reactions ${ }^{40} \mathrm{Ca}+{ }^{96} \mathrm{Zr}$ (solid line), ${ }^{40} \mathrm{Ca}+{ }^{90} \mathrm{Zr}$ (dashed line), and ${ }^{48} \mathrm{Ca}+{ }^{124} \mathrm{Sn}$ (solid line). For the reactions ${ }^{40} \mathrm{Ca}+$ ${ }^{96} \mathrm{Zr},{ }^{124} \mathrm{Sn}$, the calculated capture cross sections without the neutron transfer process are shown by dotted lines. The experimental data (symbols) are from Refs. [11, 12].

Figure 2. The same as in Fig. 1, for the reactions ${ }^{58,64} \mathrm{Ni}+{ }^{132} \mathrm{Sn}$ (solid lines) and ${ }^{58,64} \mathrm{Ni}+{ }^{130} \mathrm{Te}$ (dashed lines). The experimental data (symbols) are from Refs. $[13,14]$. For the reactions ${ }^{58,64} \mathrm{Ni}+$ ${ }^{132} \mathrm{Sn}$ (dotted lines) and ${ }^{58,64} \mathrm{Ni}+{ }^{130} \mathrm{Te}$ (dash-dotted lines), the calculated capture cross sections without the neutron transfer are shown.

transformations:

$$
E_{\text {c.m. }} \rightarrow x=\frac{E_{\text {c.m. }}-V_{b}}{\hbar \omega_{b}}, \quad \sigma_{c a p} \rightarrow \sigma_{c a p}^{r e d}=\frac{2 E_{\text {c.m. }}}{\hbar \omega_{b} R_{b}^{2}} \sigma_{c a p},
$$

where $\sigma_{c a p}=\sigma_{c a p}\left(E_{\mathrm{c} . \mathrm{m}}\right)$ is the capture cross section at bombarding energy $E_{\mathrm{c} . \mathrm{m} .}$. The frequency $\omega_{b}=\sqrt{V^{\prime \prime}\left(R_{b}\right) / \mu}$ is related to the second derivative $V^{\prime \prime}\left(R_{b}\right)$ of the total nucleus-nucleus potential $V(R)$ (the Coulomb + nuclear parts) at the barrier position $R_{b}$. With these replacements we compared the reduced calculated capture (fusion) cross sections $\sigma_{\text {cap }}^{\text {red }}$ for the reactions ${ }^{40,48} \mathrm{Ca}+{ }^{124,132} \mathrm{Sn}$ (Fig. 3). The choice of the projectile-target combination is crucial, and for the systems studied one can make unambiguous statements regarding the neutron transfer process with a positive $Q$-value when the interacting nuclei are double magic or semi-magic spherical nuclei. In this case one can disregard the strong direct nuclear deformation effects. In Fig. 3, one can see that the reduced capture cross sections 

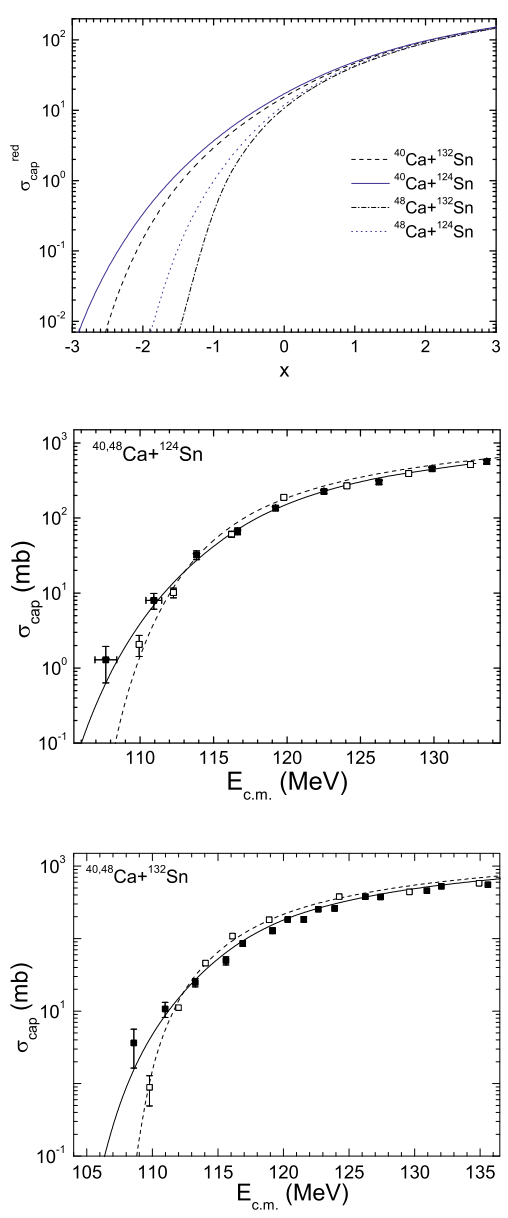

Figure 3. (Color online) The calculated reduced capture cross sections versus $\left(E_{\text {c.m. }}-V_{b}\right) /\left(\hbar \omega_{b}\right)$ in the reactions ${ }^{40} \mathrm{Ca}+{ }^{124} \mathrm{Sn}$ (solid line), ${ }^{48} \mathrm{Ca}+{ }^{124} \mathrm{Sn}$ (dashed line), ${ }^{48} \mathrm{Ca}+{ }^{124} \mathrm{Sn}$ (dotted line), and ${ }^{48} \mathrm{Ca}+{ }^{132} \mathrm{Sn}$ (dash-dotted line).
Figure 4. (Color online) The calculated capture cross sections versus $E_{\text {c.m. }}$ for the reactions ${ }^{40} \mathrm{Ca}+{ }^{124} \mathrm{Sn}$ (solid line) and ${ }^{48} \mathrm{Ca}+{ }^{124} \mathrm{Sn}$ (dashed line). The experimental data for the reactions ${ }^{40} \mathrm{Ca}+{ }^{124} \mathrm{Sn}$ (solid squares) and ${ }^{48} \mathrm{Ca}+{ }^{124} \mathrm{Sn}$ (open squares) are from Ref. [16]. In the calculations the barriers were adjusted to the experimental values.

Figure 5. (Color online) The calculated capture cross sections versus $E_{\text {c.m. }}$ for the reactions ${ }^{40} \mathrm{Ca}+{ }^{132} \mathrm{Sn}$ (solid line) and ${ }^{48} \mathrm{Ca}+{ }^{132} \mathrm{Sn}$ (dashed line). The experimental data for the reactions ${ }^{40} \mathrm{Ca}+{ }^{132} \mathrm{Sn}$ (solid squares) and ${ }^{48} \mathrm{Ca}+{ }^{132} \mathrm{Sn}$ (open squares) are from Ref. [16]. In the calculations the barriers were adjusted to the experimental values.

in the reactions ${ }^{40} \mathrm{Ca}+{ }^{124,132} \mathrm{Sn}$ with positive $Q_{2 n}$-values strongly deviate from those in the reactions ${ }^{48} \mathrm{Ca}+{ }^{124,132} \mathrm{Sn}$, where the neutron transfers are suppressed because of the negative $Q$-values. After two-neutron transfer in the reactions ${ }^{40} \mathrm{Ca}\left(\beta_{2}=0\right)+{ }^{124} \mathrm{Sn}\left(\beta_{2}=0.1\right) \rightarrow{ }^{42} \mathrm{Ca}\left(\beta_{2}=0.25\right)+{ }^{122} \mathrm{Sn}\left(\beta_{2}=\right.$ $0.1)\left(Q_{2 n}=5.4 \mathrm{MeV}\right)$ and ${ }^{40} \mathrm{Ca}\left(\beta_{2}=0\right)+{ }^{132} \mathrm{Sn}\left(\beta_{2}=0\right) \rightarrow{ }^{42} \mathrm{Ca}\left(\beta_{2}=0.25\right)+{ }^{130} \mathrm{Sn}\left(\beta_{2}=0\right)\left(Q_{2 n}=7.3\right.$ $\mathrm{MeV})$ the deformation of the light nucleus increases and the mass asymmetry of the system decreases and, thus, the value of the Coulomb barrier decreases and the capture cross section becomes larger (Fig. 3). So, because of the transfer effect, the systems ${ }^{40} \mathrm{Ca}+{ }^{124,132} \mathrm{Sn}$ show large sub-barrier enhancements with respect to the systems ${ }^{48} \mathrm{Ca}+{ }^{124,132} \mathrm{Sn}$. We observe that the $\sigma_{\text {cap }}^{\text {red }}$ in the ${ }^{40} \mathrm{Ca}+{ }^{124} \mathrm{Sn}$ $\left({ }^{48} \mathrm{Ca}+{ }^{124} \mathrm{Sn}\right)$ reaction are larger than those in the ${ }^{40} \mathrm{Ca}+{ }^{132} \mathrm{Sn}\left({ }^{48} \mathrm{Ca}+{ }^{132} \mathrm{Sn}\right)$ reaction. The reason for that is the nonzero quadrupole deformation of the heavy nucleus ${ }^{124} \mathrm{Sn}$. It should be stressed that there are almost no differences between $\sigma_{\text {cap }}^{\text {red }}$ in the reactions ${ }^{40,48} \mathrm{Ca}+{ }^{124,132} \mathrm{Sn}$ at energies above the Coulomb barrier.

In Figs. 4 and 5 one can see a good agreement between the calculated results and the experimental data in the reactions ${ }^{40,48} \mathrm{Ca}+{ }^{124,132} \mathrm{Sn}$. This means that the observed capture enhancements in the reactions ${ }^{40} \mathrm{Ca}+{ }^{124,132} \mathrm{Sn}$ at sub-barrier energies are related to the two-neutron transfer effect. Note that the slope of the excitation function strongly depends on the deformations of the interacting nuclei and, respectively, on the neutron transfer effect. 
To describe the reactions ${ }^{40,48} \mathrm{Ca}+{ }^{132} \mathrm{Sn}$ and ${ }^{48} \mathrm{Ca}+{ }^{124,132} \mathrm{Sn}$ (Figs. 4 and 5), we extracted the values of the corresponding Coulomb barrier $V_{b}$ for the spherical nuclei. There are differences between the calculated and extracted $V_{b}$. From the direct calculations of the nucleus-nucleus potentials (with the same set of parameters), we obtained $V_{b}\left({ }^{40} \mathrm{Ca}+{ }^{124} \mathrm{Sn}\right)-V_{b}\left({ }^{48} \mathrm{Ca}+{ }^{124} \mathrm{Sn}\right)=2.3 \mathrm{MeV}, \quad V_{b}\left({ }^{40} \mathrm{Ca}+{ }^{132} \mathrm{Sn}\right)-V_{b}\left({ }^{48} \mathrm{Ca}+{ }^{132} \mathrm{Sn}\right)=2.2 \mathrm{MeV}$, $V_{b}\left({ }^{40} \mathrm{Ca}+{ }^{124} \mathrm{Sn}\right)-V_{b}\left({ }^{40} \mathrm{Ca}+{ }^{132} \mathrm{Sn}\right)=1.3 \mathrm{MeV}$, and $V_{b}\left({ }^{48} \mathrm{Ca}+{ }^{124} \mathrm{Sn}\right)-V_{b}\left({ }^{48} \mathrm{Ca}+{ }^{132} \mathrm{Sn}\right)=1.2 \mathrm{MeV}$. From the extractions, we got $V_{b}\left({ }^{40} \mathrm{Ca}+{ }^{124} \mathrm{Sn}\right)-V_{b}\left({ }^{48} \mathrm{Ca}+{ }^{124} \mathrm{Sn}\right)=1.1 \mathrm{MeV}, V_{b}\left({ }^{40} \mathrm{Ca}+{ }^{132} \mathrm{Sn}\right)-V_{b}\left({ }^{48} \mathrm{Ca}+{ }^{132} \mathrm{Sn}\right)=1.0$ $\mathrm{MeV}, V_{b}\left({ }^{40} \mathrm{Ca}+{ }^{124} \mathrm{Sn}\right)-V_{b}\left({ }^{40} \mathrm{Ca}+{ }^{132} \mathrm{Sn}\right)=-0.3 \mathrm{MeV}$, and $V_{b}\left({ }^{48} \mathrm{Ca}+{ }^{124} \mathrm{Sn}\right)-V_{b}\left({ }^{48} \mathrm{Ca}+{ }^{132} \mathrm{Sn}\right)=-0.4 \mathrm{MeV}$, which seem to be unrealistically small. However, these differences of $V_{b}$ do not influence the slopes of the excitation functions but only lead to a shift of the energy scale. With a realistic isospin trend for $V_{b}, \sigma_{c a p}\left({ }^{40} \mathrm{Ca}+{ }^{124} \mathrm{Sn}\right)<\sigma_{c a p}\left({ }^{48} \mathrm{Ca}+{ }^{124} \mathrm{Sn}\right)$ and $\sigma_{c a p}\left({ }^{40} \mathrm{Ca}+{ }^{132} \mathrm{Sn}\right)<\sigma_{c a p}\left({ }^{48} \mathrm{Ca}+{ }^{132} \mathrm{Sn}\right)$ at energies above the corresponding Coulomb barriers.

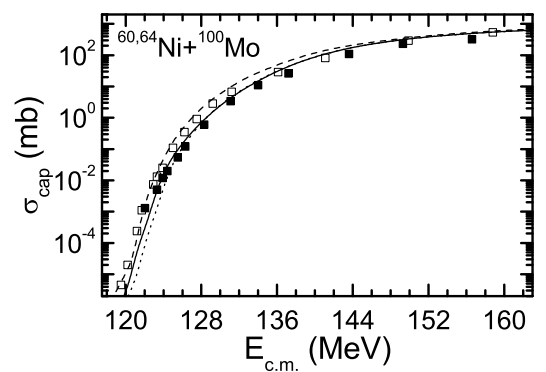

Figure 6. (Color online) The same as in Fig. 1, for the indicated reactions ${ }^{60} \mathrm{Ni}+{ }^{100} \mathrm{Mo},{ }^{150} \mathrm{Nd}$ (solid lines), and ${ }^{64} \mathrm{Ni}+{ }^{100} \mathrm{Mo},{ }^{150} \mathrm{Nd}$ (dashed lines). For the reactions ${ }^{60} \mathrm{Ni}+{ }^{100} \mathrm{Mo}$ and ${ }^{60} \mathrm{Ni}+{ }^{150} \mathrm{Nd}$, the calculated capture cross sections without the neutron transfer

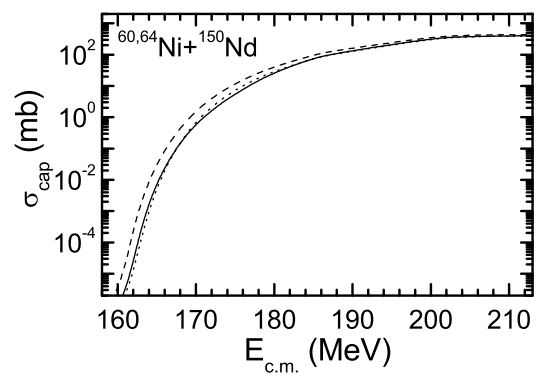
are shown by dotted lines. The experimental data for the reactions ${ }^{60} \mathrm{Ni}+{ }^{100} \mathrm{Mo}$ (closed squares) and ${ }^{64} \mathrm{Ni}+{ }^{100} \mathrm{Mo}$ (open squares) are from Ref. [17].

One can find reactions with positive $Q$-values of the two-neutron transfer where the transfer weakly influences or even suppresses the capture process. This happens if after the transfer the deformations of the nuclei do not change much or even decrease. For instance, in the reactions ${ }^{60} \mathrm{Ni}\left(\beta_{2} \approx 0.1\right)+{ }^{100} \mathrm{Mo}\left(\beta_{2}=0.231\right) \rightarrow{ }^{62} \mathrm{Ni}\left(\beta_{2}=0.198\right)+{ }^{98} \mathrm{Mo}\left(\beta_{2}=0.168\right)\left(Q_{2 n}=4.2 \mathrm{MeV}\right)$, ${ }^{64} \mathrm{Ni}\left(\beta_{2} \approx 0.087\right)+{ }^{100} \mathrm{Mo}\left(\beta_{2}=0.231\right) \rightarrow{ }^{66} \mathrm{Ni}\left(\beta_{2}=0.158\right)+{ }^{98} \mathrm{Mo}\left(\beta_{2}=0.168\right)\left(Q_{2 n}=0.94 \mathrm{MeV}\right)$, and ${ }^{60} \mathrm{Ni}\left(\beta_{2} \approx 0.1\right)+{ }^{150} \mathrm{Nd}\left(\beta_{2}=0.285\right) \rightarrow{ }^{62} \mathrm{Ni}\left(\beta_{2}=0.198\right)+{ }^{148} \mathrm{Nd}\left(\beta_{2}=0.204\right)\left(Q_{2 n}=6 \mathrm{MeV}\right)$ we expect a weak dependence of the capture cross section on the neutron transfer (Fig. 6). There is experimental evidence [17] for such an effect for the ${ }^{60} \mathrm{Ni}+{ }^{100} \mathrm{Mo}$ reaction. So, the two-neutron transfer channel with large positive $Q_{2 n}$-value weakly influences the fusion (capture) cross section. The reduced capture cross sections in the reactions ${ }^{60} \mathrm{Ni}+{ }^{100} \mathrm{Mo},{ }^{150} \mathrm{Nd}$ are close to each other in contrast to those in the reactions ${ }^{58,64} \mathrm{Ni}+{ }^{132} \mathrm{Sn},{ }^{130} \mathrm{Te}$. The ${ }^{60} \mathrm{Ni}+{ }^{150} \mathrm{Nd}$ reaction even has a small suppression due to the neutron transfer.

Figures 7-9 show the capture excitation function for the reactions ${ }^{32,36} \mathrm{~S}+\mathrm{Pd}, \mathrm{Ru}$ as a function of the bombarding energy. One can see a relatively good agreement between the calculated results and the experimental data [18]. The $Q_{2 n}$-values for the $2 n$-transfer processes are positive (negative) for all reactions with ${ }^{32} \mathrm{~S}\left({ }^{36} \mathrm{~S}\right)$. At energies above and near the Coulomb barrier the cross 

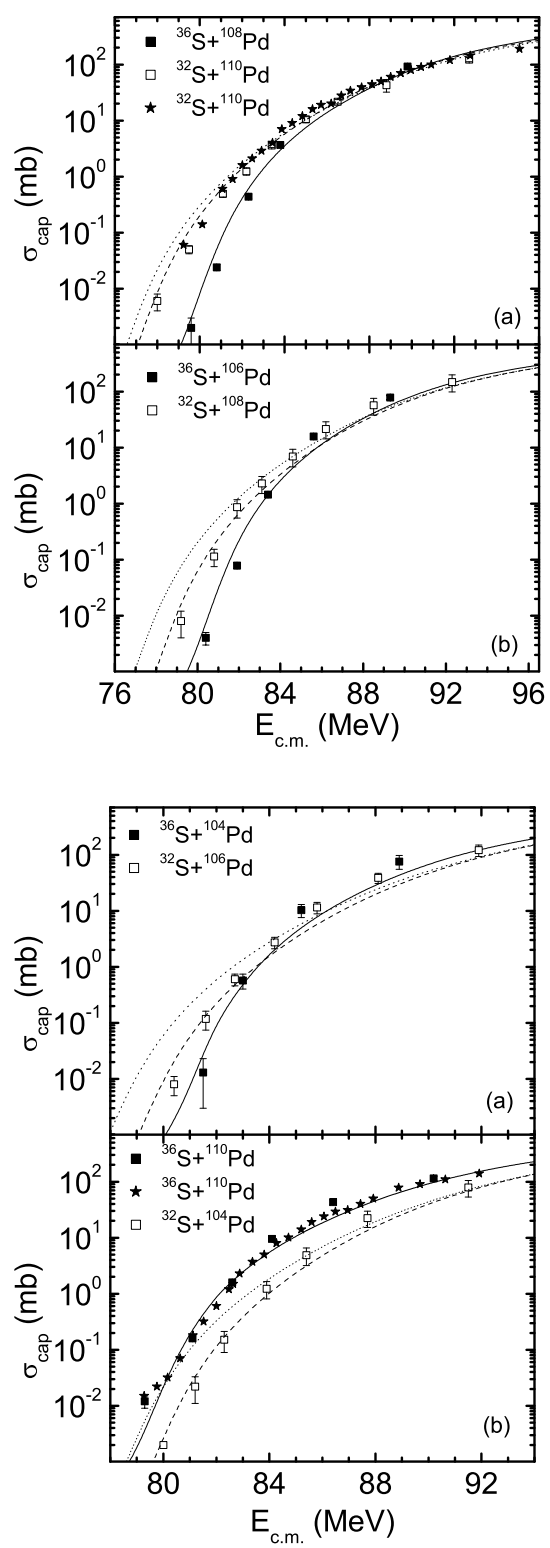

Figure 7. The calculated capture cross sections vs $E_{\text {c.m. }}$ for the reactions ${ }^{32} \mathrm{~S}+{ }^{108,110} \mathrm{Pd}$ (dashed lines) and ${ }^{36} \mathrm{~S}+{ }^{106,108} \mathrm{Pd}$ (solid lines) (a,b). For the ${ }^{32} \mathrm{~S}+{ }^{110} \mathrm{Pd}$ reaction (a), the calculated capture cross section without the neutron transfer process is shown by a dotted line. For the reaction ${ }^{32} \mathrm{~S}+{ }^{110} \mathrm{Pd}$, the experimental data from [18] and [19] are marked by open squares and stars, respectively.
Figure 8. The same as in Fig. 7, for the reactions ${ }^{32} \mathrm{~S}+{ }^{104,106} \mathrm{Pd}$ (dashed lines) and ${ }^{36} \mathrm{~S}+{ }^{104,110} \mathrm{Pd}$ (solid lines) $(\mathrm{a}, \mathrm{b})$. The dotted lines correspond to the reactions ${ }^{32} \mathrm{~S}+{ }^{104,106} \mathrm{Pd}$ when the neutron transfer is disregarded. The experimental data (symbols) are from Ref. [18].

sections with and without two-neutron transfer are almost similar. After the $2 n$-transfer (before the capture $)$ in the reactions ${ }^{32} \mathrm{~S}\left(\beta_{2}=0.312\right)+{ }^{110} \mathrm{Pd}\left(\beta_{2}=0.257\right) \rightarrow{ }^{34} \mathrm{~S}\left(\beta_{2}=0.252\right)+{ }^{108} \mathrm{Pd}\left(\beta_{2}=\right.$ $0.243),{ }^{32} \mathrm{~S}\left(\beta_{2}=0.312\right)+{ }^{108} \mathrm{Pd}\left(\beta_{2}=0.243\right) \rightarrow{ }^{34} \mathrm{~S}\left(\beta_{2}=0.252\right)+{ }^{106} \mathrm{Pd}\left(\beta_{2}=0.229\right),{ }^{32} \mathrm{~S}\left(\beta_{2}=\right.$ $0.312)+{ }^{106} \mathrm{Pd}\left(\beta_{2}=0.229\right) \rightarrow{ }^{34} \mathrm{~S}\left(\beta_{2}=0.252\right)+{ }^{104} \mathrm{Pd}\left(\beta_{2}=0.209\right),{ }^{32} \mathrm{~S}\left(\beta_{2}=0.312\right)+{ }^{104} \mathrm{Pd}\left(\beta_{2}=\right.$ $0.209) \rightarrow{ }^{34} \mathrm{~S}\left(\beta_{2}=0.252\right)+{ }^{102} \mathrm{Pd}\left(\beta_{2}=0.196\right)$, or ${ }^{32} \mathrm{~S}\left(\beta_{2}=0.312\right)+{ }^{104} \mathrm{Ru}\left(\beta_{2}=0.271\right) \rightarrow{ }^{34} \mathrm{~S}\left(\beta_{2}=\right.$ $0.252)+{ }^{102} \mathrm{Ru}\left(\beta_{2}=0.24\right),{ }^{32} \mathrm{~S}\left(\beta_{2}=0.312\right)+{ }^{102} \mathrm{Ru}\left(\beta_{2}=0.24\right) \rightarrow{ }^{34} \mathrm{~S}\left(\beta_{2}=0.252\right)+{ }^{100} \mathrm{Ru}\left(\beta_{2}=0.215\right)$, ${ }^{32} \mathrm{~S}\left(\beta_{2}=0.312\right)+{ }^{100} \mathrm{Ru}\left(\beta_{2}=0.215\right) \rightarrow{ }^{34} \mathrm{~S}\left(\beta_{2}=0.252\right)+{ }^{98} \mathrm{Ru}\left(\beta_{2}=0.195\right)$ the deformations of the nuclei decrease and the values of the corresponding Coulomb barriers increase. As a result, the transfer suppresses the capture process in these reactions at the sub-barrier energies. The suppression becomes 


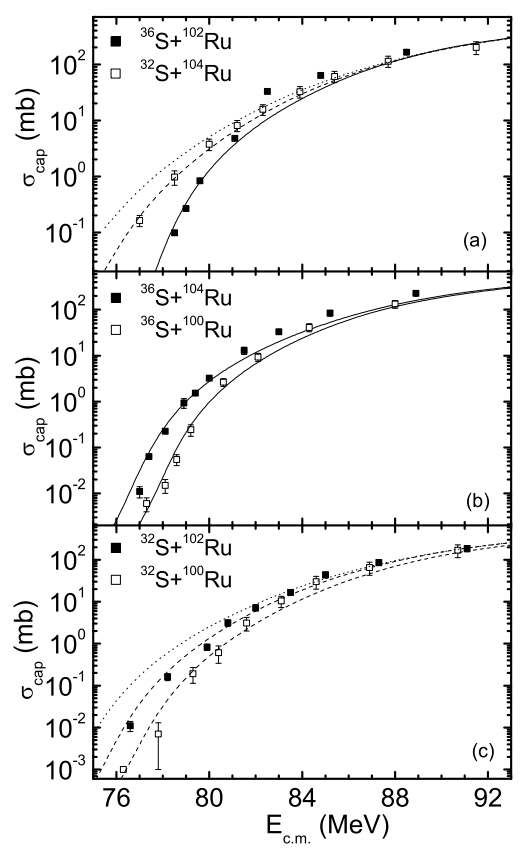

Figure 9. The calculated capture cross sections vs $E_{\text {c.m. }}$ for the reactions ${ }^{32} \mathrm{~S}+{ }^{100,102,104} \mathrm{Ru}$ (dashed lines) (a,b,c) and ${ }^{36} \mathrm{~S}+{ }^{100,102,104} \mathrm{Ru}$ (solid lines) (a,b). The dotted lines correspond to the reactions ${ }^{32} \mathrm{~S}+{ }^{102,104} \mathrm{Ru}(\mathrm{a}, \mathrm{c})$ when the neutron transfer is disregarded. The experimental data (symbols) are from Ref. [18].

stronger with decreasing energy (Figs. 7-9). As seen in Fig. 7, the capture cross sections calculated without two-neutron transfer are larger than those calculated with two-neutron transfer in the case of the ${ }^{32} \mathrm{~S}+{ }^{110} \mathrm{Pd}$ reaction. The enhancement of the sub-barrier fusion for the reactions with ${ }^{32} \mathrm{~S}$ with respect to the reactions with ${ }^{36} \mathrm{~S}$ is related to a larger deformation of ${ }^{34} \mathrm{~S}$ in comparison with ${ }^{36} \mathrm{~S}$. We observe the same behavior in the reactions ${ }^{32,36} \mathrm{~S}+{ }^{94,96,98,100} \mathrm{Mo}$.

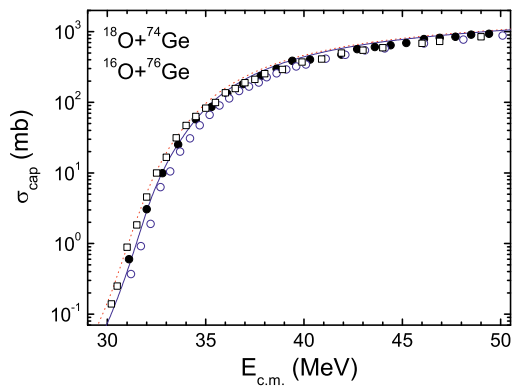

Figure 10. (Color online) The calculated (solid line) capture cross sections vs $E_{\text {c.m. }}$ for the reactions ${ }^{16} \mathrm{O}+{ }^{76} \mathrm{Ge}$ and ${ }^{18} \mathrm{O}+{ }^{74} \mathrm{Ge}$ (the curves coincide). For the ${ }^{18} \mathrm{O}+{ }^{74} \mathrm{Ge}$ reaction, the calculated capture cross sections without neutron transfer are shown by a dotted line. The experimental data for the reactions ${ }^{16} \mathrm{O}+{ }^{76} \mathrm{Ge}$ (open circles) and ${ }^{18} \mathrm{O}+{ }^{74} \mathrm{Ge}$ (open squares) are from Ref. [20]. The experimental data for the ${ }^{16} \mathrm{O}+{ }^{76} \mathrm{Ge}$ reaction (solid circles) are from Ref. [21].

Figures 10 and 11 show the excitation functions for the reactions ${ }^{18} \mathrm{O}+{ }^{74} \mathrm{Ge},{ }^{112,118,124} \mathrm{Sn}$ and ${ }^{32} \mathrm{~S}+{ }^{112,116} \mathrm{Sn}$. For the ${ }^{32} \mathrm{~S}$-induced reactions, $Q_{2 n}>0$. For the projectile ${ }^{18} \mathrm{O}$ there is a large range of positive $Q_{2 n}$-values, for example, varying from $1.4 \mathrm{MeV}$ for ${ }^{18} \mathrm{O}+{ }^{124} \mathrm{Sn}$ up to $5.5 \mathrm{MeV}$ for ${ }^{18} \mathrm{O}+{ }^{112} \mathrm{Sn}$. The agreement between the calculated results and the experimental data [20, 22] is rather good. As seen in Fig. 11, the cross sections increase systematically with the target mass number and run nearly similarly down to the lowest energy treated. In the reactions ${ }^{32} \mathrm{~S}\left(\beta_{2}=\right.$ $0.312)+{ }^{112} \mathrm{Sn}\left(\beta_{2}=0.123\right) \rightarrow{ }^{34} \mathrm{~S}\left(\beta_{2}=0.252\right)+{ }^{110} \mathrm{Sn}\left(\beta_{2}=0.122\right),{ }^{32} \mathrm{~S}\left(\beta_{2}=0.312\right)+{ }^{116} \mathrm{Sn}\left(\beta_{2}=\right.$ $0.112) \rightarrow{ }^{34} \mathrm{~S}\left(\beta_{2}=0.252\right)+{ }^{114} \mathrm{Sn}\left(\beta_{2}=0.121\right),{ }^{18} \mathrm{O}\left(\beta_{2}=0.1\right)+{ }^{74} \mathrm{Ge}\left(\beta_{2}=0.283\right) \rightarrow{ }^{16} \mathrm{O}\left(\beta_{2}=0\right)$ $+{ }^{76} \mathrm{Ge}\left(\beta_{2}=0.262\right),{ }^{18} \mathrm{O}\left(\beta_{2}=0.1\right)+{ }^{112} \mathrm{Sn}\left(\beta_{2}=0.123\right) \rightarrow{ }^{16} \mathrm{O}\left(\beta_{2}=0\right)+{ }^{114} \mathrm{Sn}\left(\beta_{2}=0.121\right)$, ${ }^{18} \mathrm{O}\left(\beta_{2}=0.1\right)+{ }^{118} \mathrm{Sn}\left(\beta_{2}=0.111\right) \rightarrow{ }^{16} \mathrm{O}\left(\beta_{2}=0\right)+{ }^{120} \mathrm{Sn}\left(\beta_{2}=0.104\right)$, and ${ }^{18} \mathrm{O}\left(\beta_{2}=0.1\right)+{ }^{124} \mathrm{Sn}\left(\beta_{2}=\right.$ 

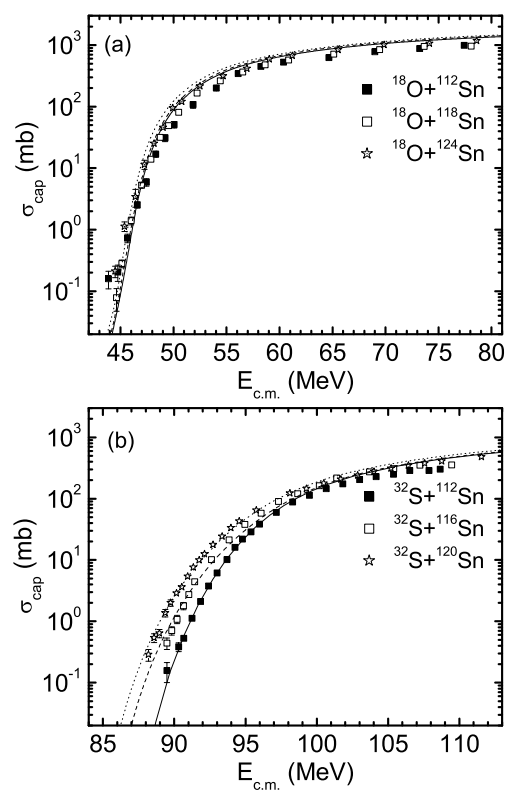

Figure 11. The calculated capture cross sections vs $E_{\text {c.m. }}$ for the reactions ${ }^{18} \mathrm{~S}+{ }^{112,118,124} \mathrm{Sn}$ (solid, dashed and dotted lines, respectively) (a) and ${ }^{32} \mathrm{~S}+{ }^{112,116,120} \mathrm{Sn}$ (solid, dashed and dotted lines, respectively) (b). The experimental data (symbols) are from Ref. [22, 23].

$0.095) \rightarrow{ }^{16} \mathrm{O}\left(\beta_{2}=0\right)+{ }^{126} \mathrm{Sn}\left(\beta_{2}=0.09\right)$ the $2 n$-transfer suppresses the capture process (Figs. 10 and 11). The sub-barrier capture cross sections for the systems ${ }^{18} \mathrm{O}+{ }^{A} \mathrm{Sn}$ studied here do not show any strong dependence on the mass number of the target isotope. Our results show that cross sections for reactions ${ }^{16} \mathrm{O}+{ }^{76} \mathrm{Ge}\left({ }^{16} \mathrm{O}+{ }^{114,120,126} \mathrm{Sn}\right)\left[Q_{2 n}<0\right]$ and ${ }^{18} \mathrm{O}+{ }^{74} \mathrm{Ge}\left({ }^{18} \mathrm{O}+{ }^{112,118,124} \mathrm{Sn}\right)$ are very similar (Fig. 10). The same behavior was observed in the recent experiments on ${ }^{16,18} \mathrm{O}+{ }^{76,74} \mathrm{Ge}[20]$.

\subsection{Neutron transfer in reactions with weakly bound nuclei}

After the neutron transfer in the reactions ${ }^{13} \mathrm{C}+{ }^{232} \mathrm{Th}\left(\beta_{2}=0.261\right) \rightarrow{ }^{14} \mathrm{C}\left(\beta_{2}=-0.36\right)+{ }^{231} \mathrm{Th}\left(\beta_{2}=\right.$ $0.261)\left(Q_{1 n}=1.74 \mathrm{MeV}\right),{ }^{15} \mathrm{C}+{ }^{232} \mathrm{Th}\left(\beta_{2}=0.261\right) \rightarrow{ }^{14} \mathrm{C}\left(\beta_{2}=-0.36\right)+{ }^{233} \mathrm{Th}\left(\beta_{2}=0.261\right)\left(Q_{1 n}=3.57\right.$ $\mathrm{MeV})$, the deformations of the target or projectile nuclei in these reactions and in the ${ }^{14} \mathrm{C}+{ }^{232} \mathrm{Th}\left(\beta_{2}=\right.$ $0.261)\left(Q_{1 n, 2 n}<0\right)$ reaction are the same. In Fig. 12 the calculated cross sections slightly increase with the mass number of $\mathrm{C}$ and are nearly parallel down to the lowest energy treated. There is a relatively good agreement between the calculated results [6] and the experimental data $[24,25]$ for the reactions ${ }^{12,13,14} \mathrm{C}+{ }^{232} \mathrm{Th}$, but the experimental enhancement of the cross section in the ${ }^{15} \mathrm{C}+{ }^{232} \mathrm{Th}$ reaction at sub-barrier energies cannot be explained by our or other [24] models. Because we take into account the neutron transfer $\left({ }^{15} \mathrm{C} \rightarrow{ }^{14} \mathrm{C}\right)$, one can suppose that this discrepancy is attributed to the influence of the breakup channel [1], which is not considered in our model. However, it is unclear why the breakup process influences only the two experimental points at the lowest energies. Different deviations of these points in energy from the calculated curve in Fig. 12 create doubt abou the influence of the breakup on the kinetic energy. So, additional experimental and theoretical investigations are desirable.

The question is whether the fusion of nuclei involving weakly bound neutrons is enhanced or suppressed at low energies. This question has been addressed in the systems ${ }^{12-15} \mathrm{C}+{ }^{208} \mathrm{~Pb}$ [26]. After the neutron transfer in the reactions ${ }^{13} \mathrm{C}+{ }^{208} \mathrm{~Pb}\left(\beta_{2}=0\right) \rightarrow{ }^{14} \mathrm{C}\left(\beta_{2}=-0.36\right)+{ }^{207} \mathrm{~Pb}\left(\beta_{2}=0\right)\left(Q_{1 n}=1.74\right.$ $\mathrm{MeV}),{ }^{15} \mathrm{C}+{ }^{208} \mathrm{~Pb}\left(\beta_{2}=0\right) \rightarrow{ }^{14} \mathrm{C}\left(\beta_{2}=-0.36\right)+{ }^{209} \mathrm{~Pb}\left(\beta_{2}=0.055\right)\left(Q_{1 n}=3.57 \mathrm{MeV}\right)$ the deformations of the light nuclei are the same as in the ${ }^{14} \mathrm{C}+{ }^{208} \mathrm{~Pb}\left(\beta_{2}=0\right)\left(Q_{1 n, 2 n}<0\right)$ reaction. The heavy nuclei are 

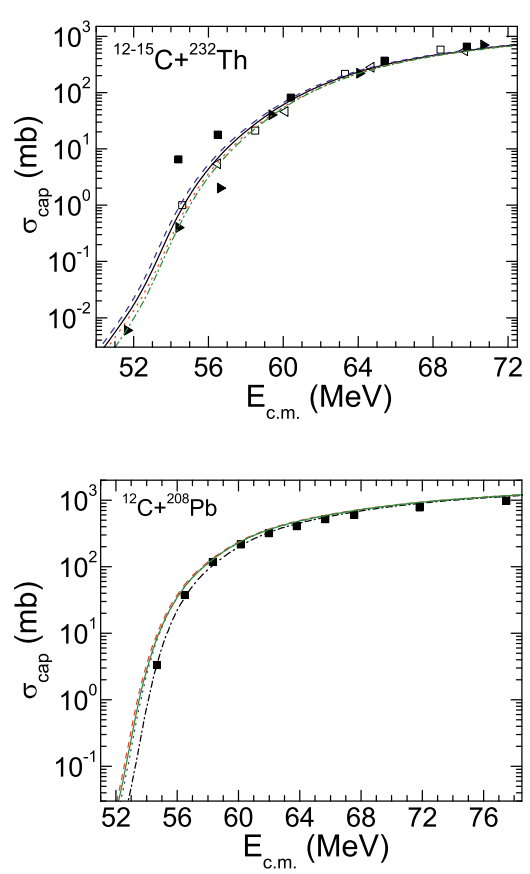

Figure 12. (Color online) The calculated (lines) and experimental (symbols) capture cross sections vs $E_{\mathrm{c} . \mathrm{m} \text {. }}$ for the reactions ${ }^{12} \mathrm{C}+{ }^{232} \mathrm{Th}$ (dash-dotted line, solid triangles), ${ }^{13} \mathrm{C}+{ }^{232} \mathrm{Th}$ (dotted line, open triangles), ${ }^{14} \mathrm{C}+{ }^{232} \mathrm{Th}$ (solid line, open squares), and ${ }^{15} \mathrm{C}+{ }^{232} \mathrm{Th}$ (dashed line, solid squares). The experimental data are from Refs. [24, 25].

Figure 13. The calculated (lines) and experimental (symbols) capture cross sections vs $E_{\text {c.m. }}$ for the reactions ${ }^{12} \mathrm{C}+{ }^{208} \mathrm{~Pb}$ (dash-dotted line), ${ }^{13} \mathrm{C}+{ }^{208} \mathrm{~Pb}$ (dotted line), ${ }^{14} \mathrm{C}+{ }^{208} \mathrm{~Pb}$ (solid line), and ${ }^{15} \mathrm{C}+{ }^{208} \mathrm{~Pb}$ (dashed line). The experimental data (solid squares) for the ${ }^{12} \mathrm{C}+{ }^{208} \mathrm{~Pb}$ reaction are from Ref. [27].

almost spherical. This means that the slopes of the excitation functions are almost the same (Fig. 13). As in the case of the ${ }^{15} \mathrm{C}+{ }^{232} \mathrm{Th}$ reaction, we do not expect enhancement of the capture cross section in the ${ }^{15} \mathrm{C}+{ }^{208} \mathrm{~Pb}$ reaction owing to the neutron transfer. The same effect was observed in Ref. [26]. The study of the reactions ${ }^{15} \mathrm{C}+{ }^{208} \mathrm{~Pb},{ }^{232} \mathrm{Th}$ at sub-barrier energies provides a good test for the verification of the effect of weakly bound nuclei on fusion and capture because it reveals the role of other effects besides neutron transfer.

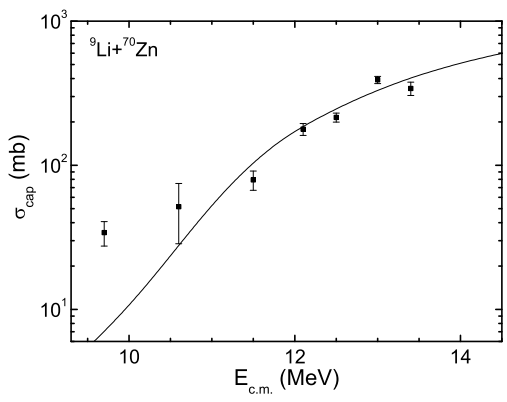

Figure 14. The calculated (solid line) and experimental (symbols) capture cross sections vs $E_{\text {c.m. }}$. for the reaction ${ }^{9} \mathrm{Li}+{ }^{70} \mathrm{Zn}$. The experimental data are from Ref. [29].

By assuming that the $2 n$-transfer process takes place and the break-up channels are closed, one can predict almost the same capture cross sections for the reaction with large positive $Q_{2 n}$ value ${ }^{6} \mathrm{He}+{ }^{206} \mathrm{~Pb}$ $\left({ }^{9} \mathrm{Li}+{ }^{68} \mathrm{Zn}\right)$ and for the complement reaction ${ }^{4} \mathrm{He}+{ }^{208} \mathrm{~Pb}\left({ }^{7} \mathrm{Li}+{ }^{70} \mathrm{Zn}\right)$. Indeed, after the transfer in the reactions ${ }^{6} \mathrm{He}+{ }^{206} \mathrm{~Pb} \rightarrow{ }^{4} \mathrm{He}\left(\beta_{2}=0\right)+{ }^{208} \mathrm{~Pb}\left(\beta_{2}=0.055\right)\left(Q_{2 n}=13.13 \mathrm{MeV}\right),{ }^{9} \mathrm{Li}+{ }^{86} \mathrm{Zn} \rightarrow{ }^{7} \mathrm{Li}\left(\beta_{2} \approx\right.$ $0.4)+{ }^{70} \mathrm{Zn}\left(\beta_{2}=0.248\right)\left(Q_{2 n}=9.60 \mathrm{MeV}\right)$, they become equivalent to the reactions ${ }^{4} \mathrm{He}+{ }^{208} \mathrm{~Pb}$ and ${ }^{7} \mathrm{Li}+{ }^{70} \mathrm{Zn}$. Therefore, the slopes of the excitation functions in the reactions with ${ }^{6} \mathrm{He}\left({ }^{9} \mathrm{Li}\right)$ and ${ }^{4} \mathrm{He}$ $\left({ }^{7} \mathrm{Li}\right)$ should be similar. This conclusion supports the experimental data of Ref. [28], where the authors 
concluded that the fusion enhancement in the ${ }^{6} \mathrm{He}+{ }^{206} \mathrm{~Pb}$ reaction (with respect to the ${ }^{4} \mathrm{He}+{ }^{208} \mathrm{~Pb}$ reaction) is rather small or absent.

By assuming that the $2 n$-transfer process occurs, we calculated the capture cross sections for the ${ }^{9} \mathrm{Li}+{ }^{70} \mathrm{Zn}$ reaction (Fig. 14). The agreement with the experimental data of Ref. [29] is quite satisfactory. At the lowest energies, the calculated cross section is a factor of $\sim 5$ smaller than the experimental value. The experimental data are well reproduced by the model [30] where two-neutron transfer from the ${ }^{70} \mathrm{Zn}$ leads to a ${ }^{11} \mathrm{Li}$ halo structure and a molecular bond between the nuclei in contact enhances the fusion cross section. Note that two-neutron transfer ${ }^{9} \mathrm{Li}+{ }^{70} \mathrm{Zn} \rightarrow{ }^{7} \mathrm{Li}+{ }^{72} \mathrm{Zn}$ with $Q_{2 n}=8.6 \mathrm{MeV}$ is energetically more favorable than the two-neutron transfer ${ }^{9} \mathrm{Li}+{ }^{70} \mathrm{Zn} \rightarrow{ }^{11} \mathrm{Li}+{ }^{68} \mathrm{Zn}$ with $Q_{2 n}=-15.4 \mathrm{MeV}$. These observations deserve further experimental and theoretical investigation including the breakup channel.

\subsection{Breakup probabilities}

The difference between the calculated capture cross section $\sigma_{c a p}^{\text {th }}$ in the absence of breakup and the experimental complete fusion cross section $\sigma_{f u s}^{\text {exp }}$ can be ascribed to the breakup effect with the probability [31]

$$
P_{\mathrm{BU}}=1-\sigma_{\text {fus }}^{\text {exp }} / \sigma_{c}^{\text {th }} \text {. }
$$

If at some energy $\sigma_{\text {fus }}^{\text {exp }}>\sigma_{c a p}^{\text {th }}$, the value of $\sigma_{c a p}^{\text {th }}$ should be normalized so as to have $P_{\mathrm{BU}} \geq 0$ at any energy. Note that $\sigma_{\text {fus }}^{e x p}=\sigma_{f u s}^{n o B U}+\sigma_{\text {fus }}^{B U}$ contains the contribution from two processes: the direct fusion of the projectile with the target $\left(\sigma_{f u s}^{n o B U}\right)$, and the breakup of the projectile followed by the fusion of the two projectile fragments with the target $\left(\sigma_{f u s}^{B U}\right)$. A more adequate estimate of the breakup probability would then be:

$$
P_{\mathrm{BU}}=1-\sigma_{\text {fus }}^{n o B U} / \sigma_{\text {cap }}^{\text {th }}
$$

which leads to larger values of $P_{\mathrm{BU}}$ than the expression we employ. However, the ratio between $\sigma_{f u s}^{n o B U}$ and $\sigma_{\text {fus }}^{B U}$ cannot be measured experimentally but can be estimated with the approach suggested in Ref. [32]. The parameters of the potential are taken to fit the height of the Coulomb barrier obtained in our calculations. The parameters of the breakup function [32] are set to describe the value of $\sigma_{\text {fus }}^{\text {exp }}$. As shown in Ref. [32] and in our calculations, in the ${ }^{8} \mathrm{Be}+{ }^{208} \mathrm{~Pb}$ reaction the fraction of $\sigma_{\text {fus }}^{B U}$ in $\sigma_{\text {fus }}^{\text {exp }}$ does not exceed a few percent at $E_{\text {c.m. }}-V_{b}<4 \mathrm{MeV}$. This fraction rapidly increases and reaches about $12-20 \%$, depending on the reaction, at $E_{\text {c.m. }}-V_{b} \approx 10 \mathrm{MeV}$. Because we are mainly interested in the energies near and below the barrier, the estimated $\sigma_{f u s}^{B U}$ does not exceed $20 \%$ of $\sigma_{\text {fus }}^{\text {exp }}$ at $E_{\mathrm{c} . \mathrm{m} .}-V_{b}<10$ $\mathrm{MeV}$. The results for $P_{\mathrm{BU}}$ are presented, taking $\sigma_{f u s}^{n o B U}$ into account, in Eq. (4).

As seen in Figs. 15 and 16, at energies above the Coulomb barriers the values of $P_{\mathrm{BU}}$ vary from 0 to $84 \%$. In the reactions ${ }^{9} \mathrm{Be}+{ }^{144} \mathrm{Sm},{ }^{208} \mathrm{~Pb},{ }^{209} \mathrm{Bi}$ the value of $P_{\mathrm{BU}}$ increases with the charge number of the target at $E_{\text {c.m. }}-V_{b}>3 \mathrm{MeV}$. This was also noted in Ref. [33]. However, the reactions ${ }^{9} \mathrm{Be}+{ }^{89} \mathrm{Y},{ }^{124} \mathrm{Sn}$ are out of this systematics. In the reactions ${ }^{6} \mathrm{Li}+{ }^{144} \mathrm{Sm},{ }^{198} \mathrm{Pt},{ }^{209} \mathrm{Bi}$ the value of $P_{\mathrm{BU}}$ decreases with increasing charge number of the target at $E_{\text {c.m. }}-V_{b}>3 \mathrm{MeV}$. While in the reactions ${ }^{9} \mathrm{Be}+{ }^{89} \mathrm{Y},{ }^{144} \mathrm{Sm},{ }^{208} \mathrm{~Pb},{ }^{209} \mathrm{Bi}$ the value of $P_{\mathrm{BU}}$ has a minimum at $E_{\mathrm{c} . \mathrm{m} .}-V_{b} \approx 0$ and a maximum at $E_{\text {c.m. }}-V_{b} \approx-(1-3) \mathrm{MeV}$, in the ${ }^{9} \mathrm{Be}+{ }^{124} \mathrm{Sn}$ reaction the value of $P_{\mathrm{BU}}$ steadily decreases with energy. In the reactions ${ }^{6} \mathrm{Li}+{ }^{144} \mathrm{Sm},{ }^{198} \mathrm{Pt},{ }^{209} \mathrm{Bi},{ }^{7} \mathrm{Li}+{ }^{208} \mathrm{~Pb},{ }^{209} \mathrm{Bi}$, and ${ }^{9} \mathrm{Li}+{ }^{208} \mathrm{~Pb}$ there is maximum of $P_{\mathrm{BU}}$ at $E_{\text {c.m. }}-V_{b} \approx-(0-1) \mathrm{MeV}$. However, in the reactions ${ }^{6} \mathrm{Li}+{ }^{208} \mathrm{~Pb}$ and ${ }^{7} \mathrm{Li}+{ }^{165} \mathrm{Ho} P_{\mathrm{BU}}$, has a minima $E_{\text {c.m. }}-V_{b} \approx 2 \mathrm{MeV}$ and no maxima at $E_{\text {c.m. }}-V_{b} \approx 0$. For ${ }^{9} \mathrm{Be}$, the breakup threshold is slightly larger than for ${ }^{6} \mathrm{Li}$. Therefore, we cannot explain a larger breakup probability at smaller $E_{\text {c.m. }}-V_{b}$ in the case of ${ }^{9} \mathrm{Be}$. 
EPJ Web of Conferences
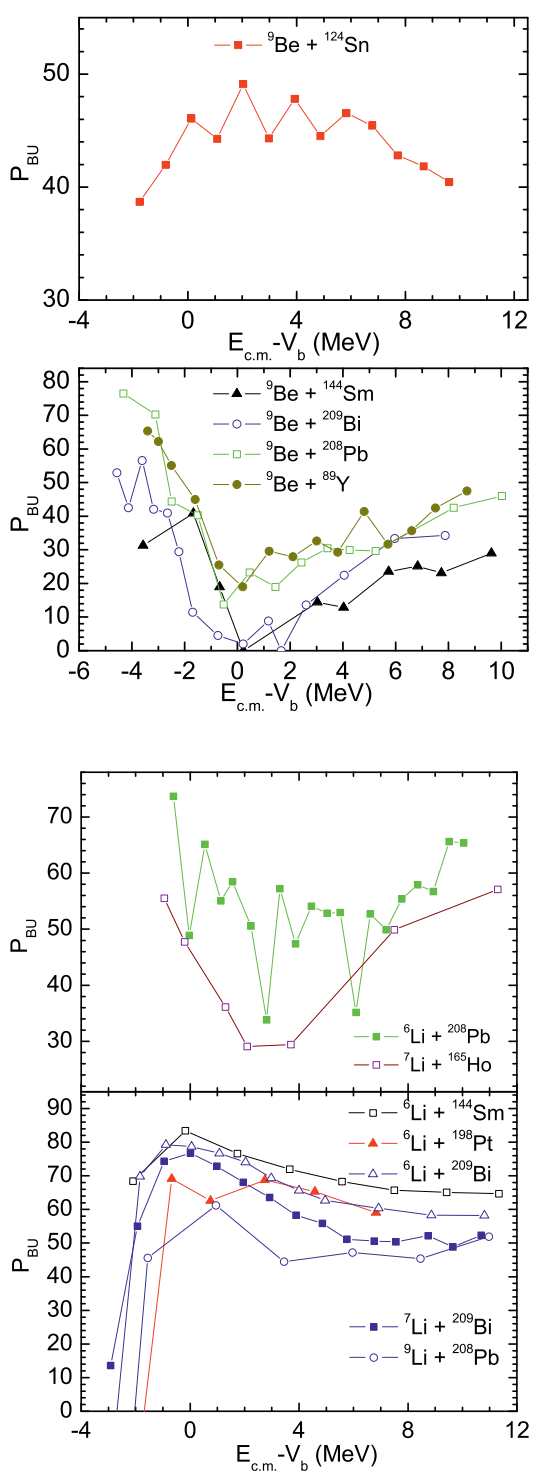

Figure 15. (Color online) The dependence of the extracted breakup probability $P_{B U}$ (in \%) vs $E_{c . m}$. $-V_{b}$ for the indicated reactions with ${ }^{9}$ Be-projectiles. Formula (4) was used.
Figure 16. (Color online) The same as in Fig. 15, but for the indicated reactions with ${ }^{6,7,9} \mathrm{Li}$-projectiles.

\section{Quasi-elastic and elastic backscattering - tools for search of breakup process in reactions with weakly bound projectiles}

The lack of a clear systematic behavior of the complete fusion suppression as a function of the target charge requires new additional experimental and theoretical studies. The quasi-elastic backscattering has been used [31, 34] as an alternative to investigate fusion (capture) barrier distributions, since this process is complementary to fusion. Since the quasi-elastic experiments are usually not as complex as the capture (fusion) and breakup measurements, they are well suited to survey the breakup probability. There is a direct relationship between the capture, the quasi-elastic scattering and the breakup processes, since any loss from the quasi-elastic and breakup channel contributes directly to capture 
(the conservation of the total reaction flux):

$$
P_{q e}\left(E_{\mathrm{c} . \mathrm{m} .}, J\right)+P_{c a p}\left(E_{\mathrm{c} . \mathrm{m} .}, J\right)+P_{B U}\left(E_{\mathrm{c} . \mathrm{m} .}, J\right)=1,
$$

where $P_{q e}$ is the reflection quasi-elastic probability, $P_{B U}$ is the breakup probability, and $P_{c a p}$ is the capture probability. The quasi-elastic scattering $\left(P_{q e}\right)$ is the sum of all direct reactions, which include elastic $\left(P_{e l}\right)$, inelastic $\left(P_{i n}\right)$, and few-nucleon transfer $\left(P_{t r}\right)$ processes. In Eq. $(5)$ we neglect the deep inelastic collision process, since we are concerned with low energies. Equation (5) can be rewritten as

$$
\frac{P_{q e}\left(E_{\mathrm{c} . \mathrm{m} .}, J\right)}{1-P_{B U}\left(E_{\mathrm{c} . \mathrm{m} .}, J\right)}+\frac{P_{c a p}\left(E_{\mathrm{c} . \mathrm{m} .}, J\right)}{1-P_{B U}\left(E_{\mathrm{c} . \mathrm{m} .}, J\right)}=P_{q e}^{n o B U}\left(E_{\mathrm{c} . \mathrm{m} .}, J\right)+P_{c a p}^{n o B U}\left(E_{\mathrm{c} . \mathrm{m} .}, J\right)=1,
$$

where

$$
P_{q e}^{n o B U}\left(E_{\mathrm{c} . \mathrm{m} .}, J\right)=\frac{P_{q e}\left(E_{\mathrm{c} . \mathrm{m} .}, J\right)}{1-P_{B U}\left(E_{\mathrm{c} . \mathrm{m} .}, J\right)}
$$

and

$$
P_{c a p}^{n o B U}\left(E_{\mathrm{c} . \mathrm{m} .}, J\right)=\frac{P_{c a p}\left(E_{\mathrm{c} . \mathrm{m} .}, J\right)}{1-P_{B U}\left(E_{\mathrm{c} . \mathrm{m} .}, J\right)}
$$

are the quasi-elastic and capture probabilities, respectively, in the absence of the breakup process. From these expressions we obtain the useful formulas

$$
\frac{P_{q e}\left(E_{\mathrm{c} . \mathrm{m} .}, J\right)}{P_{c a p}\left(E_{\mathrm{c} . \mathrm{m} .}, J\right)}=\frac{P_{q e}^{n o B U}\left(E_{\mathrm{c} . \mathrm{m} .}, J\right)}{P_{c a p}^{n o B U}\left(E_{\mathrm{c} . \mathrm{m} .}, J\right)}=\frac{P_{q e}^{n o B U}\left(E_{\mathrm{c} . \mathrm{m} .}, J\right)}{1-P_{q e}^{n o B U}\left(E_{\mathrm{c} . \mathrm{m} .}, J\right)}=a .
$$

Using Eqs. (5) and (7), we obtain the relationship between breakup and quasi-elastic processes:

$$
P_{B U}\left(E_{\text {c.m. }}, J\right)=1-P_{q e}\left(E_{\text {c.m. }}, J\right)[1+1 / a]=1-\frac{P_{q e}\left(E_{\text {c.m. }}, J\right)}{P_{q e}^{n o B U}\left(E_{\text {c.m. }}, J\right)} .
$$

The reflection quasi-elastic probability $P_{q e}\left(E_{\text {c.m. }}, J=0\right)=d \sigma_{q e} / d \sigma_{R u}$ for bombarding energy $E_{\text {c.m. }}$ and angular momentum $J=0$ is given by the ratio of the quasi-elastic differential cross section $\sigma_{q e}$ and Rutherford differential cross section $\sigma_{R u}$ at 180 degrees [34]. Employing Eq. (8) and the experimental quasi-elastic backscattering data with tightly and weakly bound isotopes-projectiles and the same compound nucleus, one can extract the breakup probability of the exotic nucleus. For example, using Eq. (8) at backward angle, the experimental $P_{q e}^{n o B U}\left[{ }^{4} \mathrm{He}+{ }^{A} \mathrm{X}\right]$ of the ${ }^{4} \mathrm{He}+{ }^{A} \mathrm{X}$ reaction with tightly bound nuclei (without breakup), and $P_{q e}\left[{ }^{6} \mathrm{He}+{ }^{A-2} \mathrm{X}\right]$ of the ${ }^{6} \mathrm{He}+{ }^{A-2} \mathrm{X}$ reaction with a weakly bound projectile (with breakup), and taking into consideration $V_{b}\left({ }^{4} \mathrm{He}+{ }^{A} \mathrm{X}\right) \approx V_{b}\left({ }^{6} \mathrm{He}+{ }^{A-2} \mathrm{X}\right)$ for the very asymmetric systems, one can extract the breakup probability of ${ }^{6} \mathrm{He}$ :

$$
P_{B U}\left(E_{\text {c.m. }}, J=0\right)=1-\frac{P_{q e}\left(E_{\text {c.m. } .}, J=0\right)\left[{ }^{6} H e+{ }^{A-2} \mathrm{X}\right]}{P_{q e}^{n o B U}\left(E_{\mathrm{c} . \mathrm{m} .}, J=0\right)\left[{ }^{4} H e+{ }^{A} \mathrm{X}\right]} .
$$

Comparing the experimental quasi-elastic backscattering cross sections in the presence and absence of breakup data in the reaction pairs ${ }^{6} \mathrm{He}+{ }^{68} \mathrm{Zn}$ and ${ }^{4} \mathrm{He}+{ }^{70} \mathrm{Zn},{ }^{6} \mathrm{He}+{ }^{122} \mathrm{Sn}$ and ${ }^{4} \mathrm{He}+{ }^{124} \mathrm{Sn}$, ${ }^{6} \mathrm{He}+{ }^{236} \mathrm{U}$ and ${ }^{4} \mathrm{He}+{ }^{238} \mathrm{U},{ }^{8} \mathrm{He}+{ }^{204} \mathrm{~Pb}$ and ${ }^{4} \mathrm{He}+{ }^{208} \mathrm{~Pb},{ }^{8} \mathrm{Li}+{ }^{207} \mathrm{~Pb}$ and ${ }^{7} \mathrm{Li}+{ }^{208} \mathrm{~Pb},{ }^{7} \mathrm{Be}+{ }^{207} \mathrm{~Pb}$ and ${ }^{10} \mathrm{Be}+{ }^{204} \mathrm{~Pb},{ }^{9} \mathrm{Be}+{ }^{208} \mathrm{~Pb}$ and ${ }^{10} \mathrm{Be}+{ }^{207} \mathrm{~Pb},{ }^{11} \mathrm{Be}+{ }^{206} \mathrm{~Pb}$ and ${ }^{10} \mathrm{Be}+{ }^{207} \mathrm{~Pb},{ }^{8} \mathrm{~B}+{ }^{208} \mathrm{~Pb}$ and ${ }^{10} \mathrm{~B}+{ }^{206} \mathrm{~Pb}$, ${ }^{8} \mathrm{~B}+{ }^{207} \mathrm{~Pb}$ and ${ }^{11} \mathrm{~B}+{ }^{204} \mathrm{~Pb},{ }^{9} \mathrm{~B}+{ }^{208} \mathrm{~Pb}$ and ${ }^{11} \mathrm{~B}+{ }^{206} \mathrm{~Pb},{ }^{15} \mathrm{C}+{ }^{204} \mathrm{~Pb}$ and ${ }^{12} \mathrm{C}+{ }^{207} \mathrm{~Pb},{ }^{15} \mathrm{C}+{ }^{206} \mathrm{~Pb}$ and ${ }^{13} \mathrm{C}+{ }^{208} \mathrm{~Pb},{ }^{15} \mathrm{C}+{ }^{207} \mathrm{~Pb}$ and ${ }^{14} \mathrm{C}+{ }^{208} \mathrm{~Pb},{ }^{17} \mathrm{~F}+{ }^{208} \mathrm{~Pb}$ and ${ }^{19} \mathrm{~F}+{ }^{206} \mathrm{~Pb}$, leading to the same corresponding compound nuclei, one can analyze the role of the breakup channels in the reactions with the light weakly bound projectiles ${ }^{6,8} \mathrm{He},{ }^{8} \mathrm{Li},{ }^{7,9,11} \mathrm{Be},{ }^{8,9} \mathrm{~B},{ }^{15} \mathrm{C}$, and ${ }^{17} \mathrm{~F}$ near and above the barrier energies. On other hand, the experimental uncertainties could probably be smaller when the same target-nucleus 
${ }^{A} \mathrm{X}$ is used in the reactions with weakly and tightly bound isotopes. Then, one can extract the breakup probability of the ${ }^{6} \mathrm{He}\left[\Delta E=V_{b}\left({ }^{4} \mathrm{He}+{ }^{A} \mathrm{X}\right)-V_{b}\left({ }^{6} \mathrm{He}+{ }^{A} \mathrm{X}\right)\right]$ :

$$
P_{B U}\left(E_{\mathrm{c} . \mathrm{m} .}, J=0\right)=1-\frac{P_{q e}\left(E_{\mathrm{c} . \mathrm{m} .}, J=0\right)\left[{ }^{6} \mathrm{He}+{ }^{A} \mathrm{X}\right]}{P_{q e}^{n o B U}\left(E_{\mathrm{c} . \mathrm{m} .}+\Delta E, J=0\right)\left[{ }^{4} \mathrm{He}+{ }^{A} \mathrm{X}\right]} .
$$

For the very asymmetric systems, one can neglect $\Delta E$.

Using the conservation of the total reaction flux, analogously one can find the following expression

$$
P_{B U}\left(E_{\mathrm{c} . \mathrm{m} .}, J\right)=1-\frac{P_{e l}\left(E_{\mathrm{c} . \mathrm{m} .}, J\right)}{P_{e l}^{n o B U}\left(E_{\mathrm{c} . \mathrm{m} .}, J\right)},
$$

which relates the breakup and elastic scattering processes. $P_{e l}^{n o B U}\left(E_{\mathrm{c} . \mathrm{m} .}, J\right)$ is the elastic scattering probability in the absence of the breakup process. So, one can extract the breakup probability of the ${ }^{6} \mathrm{He}$ at the backward angle:

$$
P_{B U}\left(E_{\mathrm{c} . \mathrm{m} .}, J=0\right)=1-\frac{P_{e l}\left(E_{\mathrm{c} . \mathrm{m} .}, J=0\right)\left[{ }^{6} \mathrm{He}+{ }^{A-2} \mathrm{X}\right]}{P_{e l}^{n o B U}\left(E_{\mathrm{c} . \mathrm{m} .}, J=0\right)\left[{ }^{4} \mathrm{He}+{ }^{A} \mathrm{X}\right]}
$$

or

$$
P_{B U}\left(E_{\mathrm{c} . \mathrm{m} .}, J=0\right)=1-\frac{P_{e l}\left(E_{\mathrm{c} . \mathrm{m} .}, J=0\right)\left[{ }^{6} \mathrm{He}+{ }^{A} \mathrm{X}\right]}{P_{e l}^{n o B U}\left(E_{\mathrm{c} . \mathrm{m} .}+\Delta E, J=0\right)\left[{ }^{4} \mathrm{He}+{ }^{A} \mathrm{X}\right]} .
$$

One concludes that the quasi-elastic or elastic backscattering technique could be a very important tool in breakup research. We propose to extract the breakup probability directly from the quasi-elastic or elastic backscattering probabilities of systems mentioned above.

\section{Summary}

The quantum diffusion approach was applied to study the role of the neutron transfer with positive $Q$-value in the capture reactions at sub-, near- and above-barrier energies. We demonstrated a good agreement of the theoretical calculations with the experimental data. We found, that the change of the magnitude of the capture cross section after the neutron transfer occurs due to the change of the deformations of nuclei. The effect of the neutron transfer is an indirect effect of the quadrupole deformation. When after the neutron transfer the deformations of nuclei do not change or slightly decrease, the neutron transfer weakly influences or suppresses the capture cross section. Good examples for this effect are the capture reactions ${ }^{60} \mathrm{Ni}+{ }^{100} \mathrm{Mo},{ }^{150} \mathrm{Nd},{ }^{18} \mathrm{O}+{ }^{64} \mathrm{Ni},{ }^{112,114,116,118,120,122,124} \mathrm{Sn},{ }^{204,206} \mathrm{~Pb}$, and ${ }^{32} \mathrm{~S}+{ }^{96} \mathrm{Zr},{ }^{94,96,98,100} \mathrm{Mo},{ }^{100,102,104} \mathrm{Ru},{ }^{104,106,108,110} \mathrm{Pd},{ }^{112,114,116,118,120,122,124} \mathrm{Sn}$. at sub-barrier energies. Thus, the general point of view that the sub-barrier capture (fusion) cross section strongly increases because of the neutron transfer with a positive $Q$-values has to be revised.

The neutron transfer effect can lead to a weak influence of halo-nuclei on the capture. Comparing the capture cross sections calculated without the breakup effect and experimental complete fusion cross sections, breakup was analyzed in reactions with weakly bound projectiles. A trend for a systematic behavior of the complete fusion suppression as a function of the target charge and bombarding energy is not achieved. The quasi-elastic or elastic backscattering was suggested to be an useful tool to study the behavior of the breakup probability.

We thank P.R.S. Gomes and A. Lépina-Szily for fruitful discussions and suggestions. This work was supported by DFG, NSFC, RFBR, and JINR grants. The IN2P3(France)-JINR(Dubna) and Polish - JINR(Dubna) Cooperation Programmes are gratefully acknowledged. 


\section{$\mathrm{CNR} * 13$}

\section{References}

[1] L.F. Canto, P.R.S. Gomes, R. Donangelo, and M.S. Hussein, Phys. Rep. 424, (2006) 1.

[2] V.V. Sargsyan, G.G. Adamian, N.V. Antonenko, and W. Scheid, Eur. Phys. J. A 45, 125 (2010).

[3] V.V. Sargsyan et al., Eur. Phys. J. A 47, 38 (2011); 48, 118 (2012); 49, 19 (2013).

[4] V.V. Sargsyan et al., Phys. Rev. C 84, 064614 (2011).

[5] V.V. Sargsyan et al., Phys. Rev. C 85, 024616 (2012); Eur. Phys. J. A 49, 54 (2013).

[6] V.V. Sargsyan et al., Phys. Rev. C 86, 014602 (2012).

[7] S. Szilner et al., Phys. Rev. C 76, 024604 (2007); S. Szilner et al., Phys. Rev. C 84, 014325 (2011); L. Corradi et al., Phys. Rev. C 84, 034603 (2011).

[8] C.H. Dasso, S. Landowne, and A. Winther, Nucl. Phys. A405, 381 (1983).

[9] G.G. Adamian, A.K. Nasirov, N.V. Antonenko, and R.V. Jolos, Phys. Part. Nucl. 25, 583 (1994);

K. Washiyama, D. Lacroix, and S. Ayik, Phys. Rev. C 79, 024609 (2009).

[10] S. Raman, C.W. Nestor, Jr, and P. Tikkanen, At. Data Nucl. Data Tables 78, 1 (2001).

[11] H. Timmers et al., Nucl. Phys. A633, 421 (1998).

[12] F. Scarlassara et al., Nucl. Phys. A672, 99 (2000).

[13] Z. Kohley et al., Phys. Rev. Lett. 107, 202701 (2011).

[14] J.F. Liang et al., Phys. Rev. C 78, 047601 (2008).

[15] L.F. Canto et al., Nucl. Phys. A821, 51 (2009); J. Phys. G 36, 015109 (2009).

[16] J.J. Kolata et al., Phys. Rev. C 85, 054603 (2012).

[17] F. Scarlassara et al., EPJ Web Conf. 17, 05002 (2011).

[18] R. Pengo et al., Nucl. Phys. A411, 255 (1983).

[19] A.M. Stefanini et al., Phys. Rev. C 52, R1727 (1995).

[20] H.M. Jia et al., Phys. Rev. C 86, 044621 (2012).

[21] E.F. Aguilera, J.J. Kolata, and R.J. Tighe, Phys. Rev. C 52, 3103 (1995).

[22] P. Jacobs, Z. Fraenkel, G. Mamane, and L. Tserruya, Phys. Lett. B 175, 271 (1986).

[23] V. Tripathi et al., Phys. Rev. C 65, 014614 (2001).

[24] M. Alcorta et al., Phys. Rev. Lett. 106, 172701 (2011).

[25] J.C. Mein et al., Phys. Rev. C 55, R995 (1997).

[26] N. Keeley and N. Alamanos, Phys. Rev. C 75, 054610 (2007).

[27] A. Mukherjee et al., Phys. Rev. C 75, 044608 (2007).

[28] R. Wolski et al., Eur. Phys. J. A 47, 111 (2011).

[29] W. Loveland et al., Phys. Rev. C 74, 064609 (2006); A.M. Vinodkumar et al., Phys. Rev. C 80, 054609 (2009).

[30] B. Balantekin and G. Kocak, AIP Conf. Proc. 1072, 289 (2008).

[31] V.V. Sargsyan et al., Phys. Rev. C 86, 054610 (2012).

[32] A. Diaz-Torres, J. Phys. G 37, 075109 (2010); Comp. Phys. Comm. 182, 1100 (2011).

[33] P.R.S. Gomes et al., Phys. Rev. C 84, 014615 (2011); P.R.S. Gomes, J. Lubian, and L.F. Canto, Phys. Rev. C 79, 027606 (2009).

[34] H. Timmers et al., Nucl. Phys. A584, 190 (1995); H.Q. Zhang et al., Phys. Rev. C 57, R1047 (1998); A.A. Sonzogni et al., Phys. Rev. C 57, 722 (1998); O.A. Capurro et al., Phys. Rev. C 61, 037603 (2000); S. Santra et al., Phys. Rev. C 64, 024602 (2001); R.F. Simões et al., Phys. Lett. B 527, 187 (2002); S. Sinha et al., Phys. Rev. C 64, 024607 (2001); E. Piasecki et al., Phys. Rev. C 65, 054611 (2002). 